\title{
Alpha entrainment drives pain relief using visual stimulation in a sample of chronic pain patients. A proof-of-concept controlled study.
}

Karen Lopez-Diaz ${ }^{1}$, James Henshaw ${ }^{1}$, Alexander J. Casson ${ }^{2}$, Christopher A. Brown $^{3,1}$, Jason R. Taylor ${ }^{1}$, Nelson J. Trujillo-Barreto ${ }^{1}$, Laura J. Arendsen ${ }^{4}$, Anthony K. P. Jones ${ }^{1}$, Manoj Sivan ${ }^{1,5}$

1 Division of Neuroscience and Experimental Psychology, University of Manchester, Manchester, UK

${ }^{2}$ Department of Electrical and Electronic Engineering, University of Manchester, UK

${ }^{3}$ Department of Psychological Sciences, University of Liverpool, Liverpool, United Kingdom

${ }^{4}$ Division of Functional and Restorative Neurosurgery, Eberhart Karls University of Tübingen, Tübingen, Germany

${ }^{5}$ Leeds Institute of Rheumatology and Musculoskeletal Medicine, University of Leeds, UK

\section{Short title/ running head}

Brain alpha entrainment for chronic pain

\section{Correspondence:}

Karen Lopez-Diaz

karen.lopez-diaz@manchester.ac.uk

\section{Conflicts of Interest}

None declared

\section{Funding}

This research was funded by MRC CiC (confidence in concept), grant number MC_PC_16053 and EPSRC Fellowship EP/N006771/1

\section{Abstract:}

One-third of the population in the UK and worldwide struggle with chronic pain.

Entraining brain alpha activity through non-invasive visual stimulation has been shown to reduce experimental pain in healthy volunteers. Neural oscillations entrainment offers a potential non-invasive and non-pharmacological intervention for patients with chronic pain, which can be delivered in the home setting and has the 
potential to reduce use of medications. However, evidence supporting its use in patients with chronic pain is lacking. This study explores whether a) alpha entrainment increase alpha power in patients and b) whether this increase in alpha correlates with analgesia.

28 patients with chronic pain sat in a comfortable position and underwent 4-minute visual stimulation using customised goggles at $10 \mathrm{~Hz}$ (alpha) and $7 \mathrm{~Hz}$ (control) frequency blocks in a randomised cross-over design. 64-channel

Electroencephalography (EEG) and 11-point Numeric Rating Scale (NRS) pain intensity and pain unpleasantness scores were recorded before and after stimulation.

EEG analysis revealed frontal alpha power was significantly higher when stimulating at $10 \mathrm{~Hz}$ when compared to $7 \mathrm{~Hz}$. There was a significant positive correlation between increased frontal alpha and reduction in pain intensity $(r=0.33, p<0.05)$ and pain unpleasantness $(r=0.40, p<0.05)$ in the $10 \mathrm{~Hz}$ block.

This study provides the first proof of concept that changes in alpha power resulting from entrainment correlate with an analgesic response in patients with chronic pain. Further studies are warranted to investigate dose-response parameters and equivalence to analgesia provided by medications.

Keywords: Electroencephalography (EEG); alpha activity; treatment; visual stimulation

\section{Introduction:}

Chronic pain is a global health crisis and affects more than one-third of the population in most countries [1]. It has a profound impact on the physical, mental and social well-being of those affected [2; 3]; this is aggravated by the fact that medications currently used to treat it have limited effectiveness and a number of 
debilitating side effects $[4 ; 5]$. There is therefore an urgent need to develop new alternative, non-pharmacological, safe and effective treatments.

Recently, modulation of the brain's alpha activity (frequency range $8-12 \mathrm{~Hz}$ ) has emerged as a promising option for pain management[6]. Alpha activity recorded over frontal electrodes increases after a placebo induced expectation of pain relief in healthy volunteers [7], suggesting that increased alpha might be an indicator of pain resilience. This led to the speculation that techniques that increase alpha power might be used to alleviate pain in patients with chronic pain[8-11].

Alpha activity can be entrained in the brain by exposing the person to visual, auditory, tactile, or electrical simulation in the $10 \mathrm{~Hz}$ frequency range[12]. Entrainment refers to a phenomenon in the brain when it is presented with an external stimulation at a certain frequency. This leads the brain's oscillatory neural activity to synchronise with the external stimuli, which ultimately results in an increase of brain power at the stimulation frequency[13-16]. To date, alpha entrainment has been successful in reducing experimental pain in healthy volunteers [17-19], but only one study has demonstrated a reduction in pain intensity in patients with chronic pain associated with increased somatosensory alpha power using transcranial current stimulation [11]. To our knowledge, there are no studies so far that have used visual stimulation for frontal alpha entrainment in chronic pain patients and have shown correlation between changes in alpha power and changes in pain intensity.

\section{Methods}

The current study is an extension of a preliminary study by Arendsen [20] with eight new participants added (28 participants in total). All participants had a 
diagnosis of chronic musculoskeletal pain (pain present for at least three months). The mean age of participants was $44.68(S D=17.03) ; 20$ participants were female and 8 were male.

The study used a within-subject design and alpha stimulation $(10 \mathrm{~Hz})$ was compared to a control stimulation $(7 \mathrm{~Hz})$ in a sitting position. Participants were visually stimulated using custom-made goggles with $10 \mathrm{~Hz}$ frequency flashing lights for 5 minutes, the first minute consisted of a non-rhythmic stimulation which was used as a baseline for the remaining 4 minutes of rhythmic visual stimulation.

Behavioural response was analysed by asking participants to evaluate their pain intensity and unpleasantness using an 11-point Numerical Rating Scale (NRS) for pain intensity ( $0=$ no pain, $10=e x t r e m e$ pain) and an 11 -point NRS for pain unpleasantness $(0=$ no unpleasantness, $10=$ extreme unpleasantness). Participants were asked to rate their pain intensity and unpleasantness level before and after each stimulation condition.

EEG was recorded using $64 \mathrm{Ag} / \mathrm{AgCl}$ electrodes attached to a cap according to the extended standard 10-20 system, using the BrainCap MR, BrainAmp DC/MR amplifiers, and the EEG data recording software BrainVision Recorder (Brain Products $\mathrm{GmbH}$, Germany). The reference was set as the FCz electrode and the AFz was used as the ground electrode. EEG was recorded with a sampling rate of $500 \mathrm{~Hz}$ and band-pass filter settings of DC-100 Hz.

EEG recordings were pre-processed using the EEGLAB toolbox[21], running in MATLAB (The Mathworks, Inc., Natick, MA, MATLAB versions R2017a). Bad channels were interpolated (spherical interpolation) before re-referencing to the common average. Next high-pass $(0.05 \mathrm{~Hz})$ and low-pass $(30 \mathrm{~Hz})$ bands were 
filtered, and the continuous data were segmented into 2-second consecutive epochs to enable later visual inspection of the data. The data were decomposed into independent signals using Independent Component Analysis (ICA) to remove components reflecting artefactual sources. The Runica algorithm was used and the number of components to be calculated was adjusted for the number of interpolated channels (Number of channels - Number of interpolated channels). The median number of components that were projected out was 2.3 with a range between 1 to 6 . Components that reflected eye blinks and movement were removed as well as any large muscle artifacts.

In this analysis we looked at the spectral mass of the signal between 8 and 12 $\mathrm{Hz}$ instead of averaging across the range or using a single frequency peak. Frequency analysis was performed using the Matlab-based Fieldtrip Toolbox[22]. Average alpha power across all 2 second windows was calculated for each rhythmic visual stimulation condition (7 and $10 \mathrm{~Hz}$ ) and its corresponding baselines. It was then averaged across the electrodes corresponding to the frontal Region of Interest (ROI) (Fp1, Fp2, Fpz, AF3, AF4, F1, F2, FZ)[20] to obtain a spectrum of frontal alpha power for each condition. It was not averaged over frequency, but instead we kept the averaged alpha power for each individual frequency within this range. The spectral mass was then calculated by computing the area under the spectrum along this range $(8$ to $12 \mathrm{~Hz})$ in each case. The same was done for 7 and $10 \mathrm{~Hz}$ stimulation conditions baselines. The spectral mass of the response was then divided by the spectral mass of its corresponding baseline to eliminate any constant present and obtain a Spectral Mass Ratio (SMR) for every individual in each condition, which was then log transformed. 
Statistical analysis was performed using MATLAB. Behavioural responses were analysed for each experimental condition by comparing pain intensity and unpleasantness differences (NRS 0-10) before and after each session. A one-way ANOVA was performed to see if there was a significant difference in the log-SMR between the two stimulation frequencies. Pearson's correlations were then run between log-SMR and behavioural responses.

\section{Results}

The mean and standard deviation of the log-SMR for both stimulation conditions were: $7 \mathrm{~Hz}$ (mean: -0.2417 , std: 0.3458 ) and $10 \mathrm{~Hz}$ (mean: 0.3943 , std: 0.5571). There was a significant difference between the log-SMR observed within the two conditions $(F=26.35, p<0.01)$. The distributions of the log-SMR values obtained are shown in Figure 1.

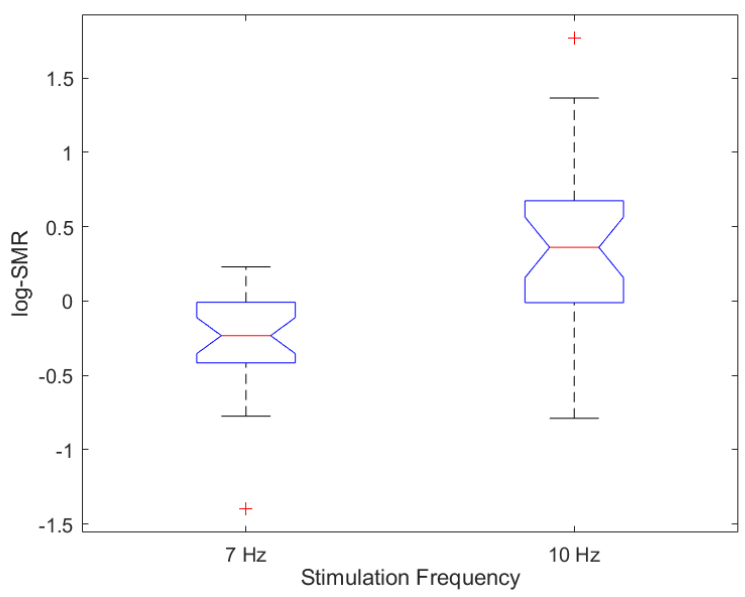

Figure 1: Boxplot of log-SMR during 7 and $10 \mathrm{~Hz}$ visual stimulation standardized against their respective baseline period, i.e. the change in frontal alpha spectral mass during stimulation compared to baseline.

The average reduction in pain intensity for $7 \mathrm{~Hz}$ stimulation was 0.38 (std:1.22) and for $10 \mathrm{~Hz}$ stimulation was 0.53 (std: 1.84). The average unpleasantness increased for $7 \mathrm{~Hz}$ stimulation $(-0.11$, std: 1.22) but there was also an average decrease in unpleasantness for $10 \mathrm{~Hz}(0.18$, std: 2.42) 
The relationships between pain intensity and unpleasantness differences to baseline, and the EEG log-SMR, are shown in Figure 2. Correlation analysis showed that in the $10 \mathrm{~Hz}$ stimulation condition, there was positive correlation between frontal alpha increase and pain reduction $(r=0.33, p<0.05)$; and also a positive correlation between frontal alpha increase and unpleasantness reduction $(r=0.40, p<0.05)$. There were no significant correlations observed between alpha power and pain intensity or unpleasantness in the $7 \mathrm{~Hz}$ stimulation condition.
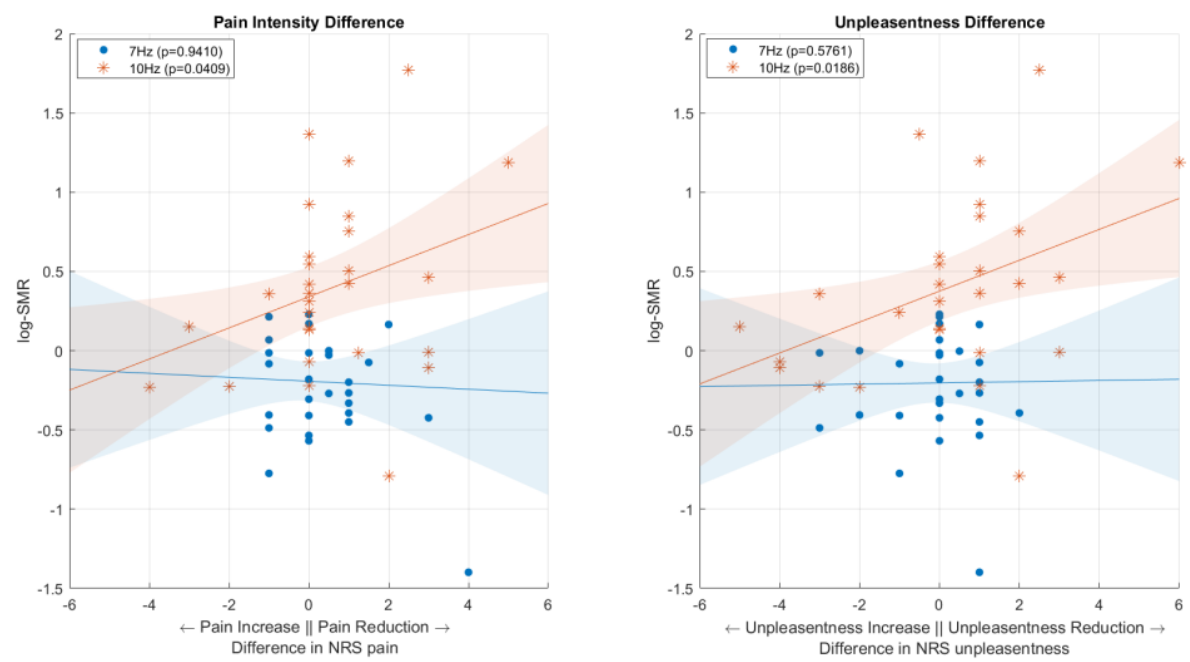

Figure 2. Scatter plot of log-SMR on the $y$-axis and the difference in pain intensity and unpleasantness ratings comparing pre- and post-stimulation for the 7 and $10 \mathrm{~Hz}$ stimulation.

\section{Discussion:}

This is the first study to show a correlation between increased frontal alpha power from visual stimulation and reduction of pain in chronic pain patients. Frontal alpha spectral mass was significantly higher during the $10 \mathrm{~Hz}$ stimulation compared to the $7 \mathrm{~Hz}$ control stimulation. It was observed that despite the brief stimulation time (4 minutes), there was a positive correlation observed between frontal alpha spectral mass and a perceived reduction in pain and unpleasantness levels.

As in previous analysis, we showed the possibility of modulating alpha activity in patients with long term chronic pain by using rhythmic visual stimulation [20]. 
Unlike previous results however, we studied modulations of the total spectral mass in the whole alpha band $(8-12 \mathrm{~Hz})$, rather than focussing on the amplitude of a specific peak within the band (usually at $10 \mathrm{~Hz}$ ). This type of analysis allowed for considering possible individual variations of the specific frequency at which the modulation occurs. Using this approach, we added to the existing literature on the therapeutic treatment of chronic pain via visual stimulation by demonstrating a specific effect in the frontal ROI.

It also builds on other studies that demonstrated that visual alpha stimulation can increase alpha power and reduce experimental pain settings [17]. An increase in frontal and somatosensory alpha power through electrical stimulation using tACS has also been associated with pain reduction [11].

The results of this exploratory study are very promising since it observes a correlational effect between increased alpha levels in the 8-12 $\mathrm{Hz}$ range and pain/unpleasantness reduction in actual chronic pain patients. The results justify further research into this field, as this procedure seems to be a promising alternative to pain medication in chronic pain relief; for example, further research is warranted focusing on optimising stimulation times (dose) and protocols.

\section{Acknowledgements:}

The authors thank Timothy Rainey of the Human Pain Research Group, University of Manchester, for his technical help and help with participants for this study

\section{Reference:}

1. Fayaz A, Croft P, Langford RM, Donaldson LJ, Jones GT. Prevalence of chronic pain in the UK: A systematic review and meta-analysis of population studies. BMJ Open 2016; 6 (6):

2. Breivik H, Collett B, Ventafridda V, Cohen R, Gallacher D. Survey of chronic 
pain in Europe: prevalence, impact on daily life, and treatment. Eur. J. Pain 2006; 10 (4): 287-333.

3. Van Hecke O, Torrance N, Smith BH. Chronic pain epidemiology and its clinical relevance. Br. J. Anaesth. 2013; 111 (1): 13-18.

4. Chou R, Turner JA, Devine EB, Hansen RN, Sullivan SD, Blazina I, et al. The effectiveness and risks of long-term opioid therapy for chronic pain: a systematic review for a National Institutes of Health Pathways to Prevention Workshop. Ann. Intern. Med. 2015; 162 (4): 276-286.

5. Turk DC. Clinical Effectiveness and Cost-Effectiveness of Treatments for Patients With Chronic Pain. Clin. J. Pain 2002; 18 (6):

6. Jensen O, Mazaheri A. Shaping functional architecture by oscillatory alpha activity: Gating by inhibition. Front. Hum. Neurosci. 2010; 4 (November): 1-8.

7. Huneke NTM, Brown CA, Burford E, Watson A, Trujillo-Barreto NJ, El-Deredy W, et al. Experimental Placebo Analgesia Changes Resting-State Alpha Oscillations. PLoS One 2013; 8 (10): 1-11.

8. Tu Y, Zhang Z, Tan A, Peng W, Hung YS, Moayedi M, et al. Alpha and gamma oscillation amplitudes synergistically predict the perception of forthcoming nociceptive stimuli. Hum. Brain Mapp. 2016; 37 (2): 501-514.

9. Babiloni C, Brancucci A, Percio C Del, Capotosto P, Arendt-Nielsen L, Chen ACN, et al. Anticipatory Electroencephalography Alpha Rhythm Predicts Subjective Perception of Pain Intensity. J. Pain 2006; 7 (10): 709-717.

10. Camfferman D, Lorimer Moseley G, Gertz K, Pettet MW, Jensen MP. Waking EEG cortical markers of chronic pain and sleepiness. Pain Med. (United States) 2017; 18 (10): 1921-1931.

11. Ahn S, Prim JH, Alexander ML, McCulloch KL, Fröhlich F. Identifying and 
Engaging Neuronal Oscillations by Transcranial Alternating Current

Stimulation in Patients With Chronic Low Back Pain: A Randomized,

Crossover, Double-Blind, Sham-Controlled Pilot Study. J. Pain 2019; 20 (3):

277.e1-277.e11.

12. Thut G, Schyns PG, Gross J. Entrainment of perceptually relevant brain oscillations by non-invasive rhythmic stimulation of the human brain. Front. Psychol. 2011; 2 (JUL): 1-10.

13. de Graaf TA, Gross J, Paterson G, Rusch T, Sack AT, Thut G. Alpha-Band Rhythms in Visual Task Performance: Phase-Locking by Rhythmic Sensory Stimulation. PLoS One 2013; 8 (3): 29-32.

14. Helfrich RF, Schneider TR, Rach S, Trautmann-Lengsfeld SA, Engel AK, Herrmann CS. Entrainment of brain oscillations by transcranial alternating current stimulation. Curr. Biol. 2014; 24 (3): 333-339.

15. Spaak E, de Lange FP, Jensen O. Local entrainment of alpha oscillations by visual stimuli causes cyclic modulation of perception. J. Neurosci. 2014; 34 (10): 3536-3544.

16. Vossen A, Gross J, Thut G. Alpha power increase after transcranial alternating current stimulation at alpha frequency (a-tACS) reflects plastic changes rather than entrainment. Brain Stimul. 2015; 8 (3): 499-508.

17. Ecsy K, Brown CA, Jones AKP. Cortical nociceptive processes are reduced by visual alpha-band entrainment in the human brain. Eur. J. Pain (United Kingdom) 2018; 22 (3): 538-550.

18. Ecsy K, Jones AKP, Brown CA. Alpha-range visual and auditory stimulation reduces the perception of pain. Eur. J. Pain (United Kingdom) 2017; 21 (3): $562-572$. 
19. Hassaan A, Jones A, Sivan M. The brain alpha rhythm in the perception and modulation of pain. Adv. Clin. Neurosci. Rehabil. 2020;

20. Arendsen LJ, Henshaw J, Brown CA, Sivan M, Taylor JR, Trujillo-Barreto NJ, et al. Entraining Alpha Activity Using Visual Stimulation in Patients With Chronic Musculoskeletal Pain: A Feasibility Study. Front. Neurosci. 2020; 14: $1-30$

21. Delorme A, Makeig S. EEGLAB: An open source toolbox for analysis of singletrial EEG dynamics including independent component analysis. J. Neurosci. Methods 2004; 134 (1): 9-21.

22. Oostenveld R, Fries P, Maris E, Schoffelen JM. FieldTrip: Open source software for advanced analysis of MEG, EEG, and invasive electrophysiological data. Comput. Intell. Neurosci. 2011; 2011: 